\section{An independent $\mathrm{CBCT}$ imaging service}

CBCT imaging is used prior to many dental procedures as it allows for a more accurate and safer patient outcome. One such procedure is the extraction of the lower third molar, due to its ability to accurately determine the relationship of the roots of the tooth with the inferior dental canal (IDC).

In many patients, the lower third molar has a very close relationship or is communicating directly with the IDC. Although this can be assessed on periapical and dental panoramic images, using $\mathrm{CBCT}$ prior to extraction can significantly reduce the risk of post-operative complications such as paraesthesia.
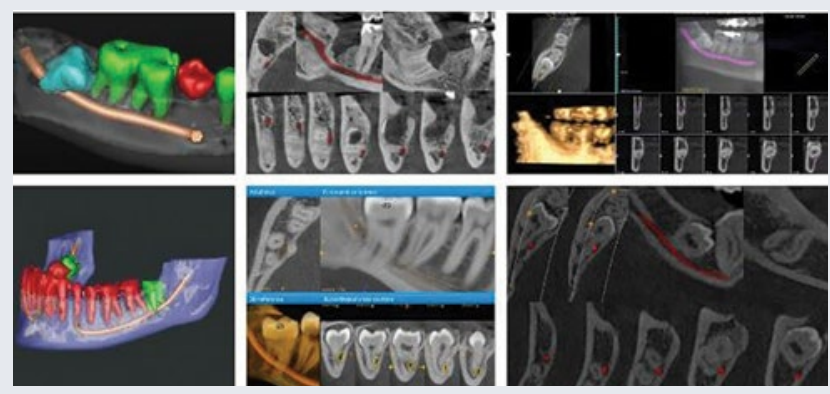

CBCT can also be used to look at the close relationship of the lower third molar with the second molar and assessing potential crown and root pathology, for example areas of decay and resorption. It can also highlight early follicular changes which can lead to dentigerous cyst development.

CT Dent has been providing an independent dental imaging service to dental practitioners since 2007. CT Dent's constant investment in new technology means those using its service receive consistently high-quality scans.

Visit www.ct-dent.co.uk or call 02074875717.

\section{Standing out from the crowd}

Rodericks Dental was proud to stand out from the crowd at the British Dental Conference and Dentistry Show, where it showed professionals what it means to be run by dentists, for dentists.

The team demonstrated what it means for them to be 'clinically-led', focusing on four core areas - clinical support, professional development, quality of all treatments and clinical efficiency.

Rodericks' own Associate Director for Professional Development, Carlos Clark, also presented two popular sessions on career development, sharing his extensive wisdom and empowering young dentists.

To find out more about why Rodericks does things differently, contact the team today.

For more information visit www.rodericksdentalcareers.co.uk.

\section{Custom-developed for innovative RPD design}

Enthusiastic about a metal-free alternative for removable partial dentures (RPD), visitors to the British Dental Conference and Dentistry Show 2019 headed for the Solvay Dental 360 stand.

Here, delegates saw the quality of digitally designed RPD frames made from Ultaire AKP - a custom-developed material that is strong, biocompatible, lightweight and taste free.

Ultaire AKP is a high performance polymer that improves the patient experience with secure retention, increased comfort and enhanced aesthetics.

If you didn't get to the show this year, but would like to know more about metal-free RPDs, contact Solvay Dental 360 now. To book a Solvay Dental 360 Professional Lunch and Learn or to find more information about Ultaire AKP and Dentivera milling discs, visit www.solvaydental360.com.

\title{
The UK's first bioplastic tongue cleaner
}

Oral health brand TePe has expanded its sustainably produced TePe GOOD range with the introduction of the TePe GOOD Compact toothbrush and TePe GOOD Tongue Cleaner, the UK's first bio-plastic tongue cleaner.

The TePe GOOD Compact toothbrush and TePe GOOD Tongue Cleaner are produced using renewable raw materials, sugar cane and castor oil. This enables TePe to recirculate up to $95 \%$ of the $\mathrm{CO}_{2}$ emissions during the products' life cycle, offering a more sustainable choice to consumers and the industry, without compromising on product quality, design or hygiene.

As with all TePe products, the TePe GOOD Compact toothbrush and TePe GOOD Tongue Cleaner are manufactured using 100\% green energy at TePe's Swedish Malmö factory, which has the largest solar power plant in the city.

In addition to its sustainability benefits, the TePe GOOD Compact toothbrush has been developed in collaboration with dental experts to ensure a high-quality design, featuring an ergonomic handle, tapered head and rounded filaments for maximum cleanliness. The TePe GOOD Compact toothbrush is recommended for children and adults who prefer a smaller brush head.
The new TePe GOOD Tongue Cleaner has been carefully designed to remove bacteria from the tongue, helping to prevent bad breath and improve oral health. It corresponds to the natural shape of the tongue, making it easier to reach far back in the mouth, while the slim shape reduces the gag reflex. What's more, with three slightly raised cleaning surfaces for a triple-effect, one stroke should be enough to clean the tongue.

The TePe GOOD Compact toothbrush and TePe GOOD Tongue Cleaner were unveiled at the British Dental Conference and Dentistry Show 2019 and will be available from the end of May 2019 from all dental wholesalers in the UK.

The TePe GOOD Compact toothbrush has a suggested retail price of $£ 2.75$, while TePe GOOD Tongue Cleaner has a recommended retail price of $£ 3.35$. For orders or more information contact your local dental wholesaler. 